\title{
POPULATION STRUCTURE IN THE SWORD PRAWN (PARAPENAEOPSIS HARDWICKII) FROM THE EAST CHINA SEA AND TAIWAN STRAIT INFERRED FROM INTRON SEQUENCES
}

\author{
Tzong-Der Tzeng, Ta-Jen Chu, Daryi Wang, Hsien-Lu Haung, and Shean-Ya Yeh
}

(T-DT, correspondent) College of Liberal Education, Shu-Te University, No. 59, Hun Shan Road, Hun Shan Village,

Yen Chau, Kaohsiung County 824, Taiwan R.O.C. (tdtzeng@mail.stu.edu.tw);

(T-JC) Department of Leisure and Recreation Management, Chung Hua University, 707 sec. 2, Wu Fu Road, Hsin Chu, Taiwan 300, R. O. C. (tajen@chu.edu.tw)

(DW) Research Center for Biodiversity, Academia Sinica, 128 Academia Road, Section 2, Nankang, Taipei 115, Taiwan R.O.C. (dywang@gate.sinica.edu.tw);

(H-LH) Department of Nutrition and Health Science, Fooyin University, 151, Chinhsueh Road, Ta-liao, Kaohsiung, Taiwan, R.O.C. (estrus@mail2000.com.tw);

(S-YY) Institute of Oceanography, National Taiwan University, P.O. Box 23-13, Taipei 106,

Taiwan, R.O.C. (sheanya@ntu.edu.tw)

\begin{abstract}
A B S T R A C T
Sequence analyses on the intron from the elongation factor- $1 \alpha$ gene were conducted to examine the population structure of sword prawn (Parapenaeopsis hardwickii) in the East China Sea and Taiwan Strait. Five samples including 207 individuals were separately collected from the north of East China Sea (NECS) and waters off Tamsui, Taichung, Putai, and Cheding, and 102 alleles were identified. Despite no phylogeographic structure in alleles, pairwise $F_{\text {ST }}$ values and analysis of molecular variance (AMOVA) showed significant genetic difference between the NECS and the other four samples. The UPGMA tree of five samples showed two distinct clusters; one included the NECS sample; the other included the rest of samples. The results suggest that two populations exist in the East China Sea and Taiwan Strait. To ensure resource sustainability and maintenance, the sword prawn in the Taiwan Strait and the north of the East China Sea should be treated as two separate populations and then be separately managed in future. Both Tajima's $D$ and Fu and Li's $D$ statistics and analysis of mismatch distribution for overall alleles suggested that sword prawn in studied area had experienced population expansion.
\end{abstract}

KeY Words: Elongation factor-1 $\alpha$ gene, East China Sea, population structure, Parapenaeopsis hardwickii, Taiwan Strait

\section{INTRODUCTION}

Information on population structure is essential for the management and conservation of genetic resources in exploited marine organisms (Hillis et al., 1996). Recent researches on population genetic structure have utilized new analytical and technical tools that provide high-resolution genetic information. These tools include polymerase chain reaction (PCR) amplification, sequencing of DNA fragments, and the phylogenetic analysis of allelic variants. Many of the data for these studies have come from sequences of mitochondrial genes. However, the analysis of mitochondrial DNA sequence variability is widely recognized as suffering from two main weaknesses. First, the mitochondrial genome is only a single genetic locus. A second problem is that such analyses on mitochondrial DNA sequence allow only the reconstruction of maternal lineages (Wilson et al., 1985). The nuclear markers would differ from mitochondrial markers in their rates of evolution and biparental mode of inheritance. The ideal nuclear marker for intraspecific studies would show relatively high levels of neutral variation. One approach to find such markers has been to target introns in highly conserved nuclear genes (Bradley and Hills, 1997). An additional benefit of such intron is that "universal" primers can be designed that will anneal to regions in the highly conserved exons flanking noncoding introns (Palumbi and Baker, 1994).
Analyses on population genetic structure of marine biota have frequently revealed that organisms with high dispersal capacity would have little genetic distinction over large geographic scales (Hellberg, 1996). These studies suggest that there are high levels of gene flow between marine populations. However, there is growing evidence that widespread marine organisms are more genetically structured than expected given their high dispersal potential and apparent lack of barriers to dispersal in the ocean (Palumbi, 1997; Benzie, 1999; Briggs, 1999). Thus, there may be limits to the actual dispersal of marine organisms with high dispersal potential (Benzie and Williams, 1997). These limits vary widely with species, habitats, local ocean conditions or historical events, and they may produce sufficient chances for genetic distinction (Palumbi, 1994).

Sword prawn, Parapenaeopsis hardwickii Miers, 1878, is distributed mainly in the Indo-West Pacific from Pakistan to Japan and lives at 5 to $90 \mathrm{~m}$ in the sand bottom areas. This species is one of the most abundant and highly valued species in the East China Sea and Taiwan Strait (Wu, 1985; Song and Ding, 1993). The life history of the sword prawn, with an offshore planktonic larval phase, an estuarine postlarval and juvenile phase, and an offshore adult and spawning phase (Dall et al., 1990), may allow moderate gene flow among populations. Two morphologically distinguishable populations of sword prawn in the East China Sea and 


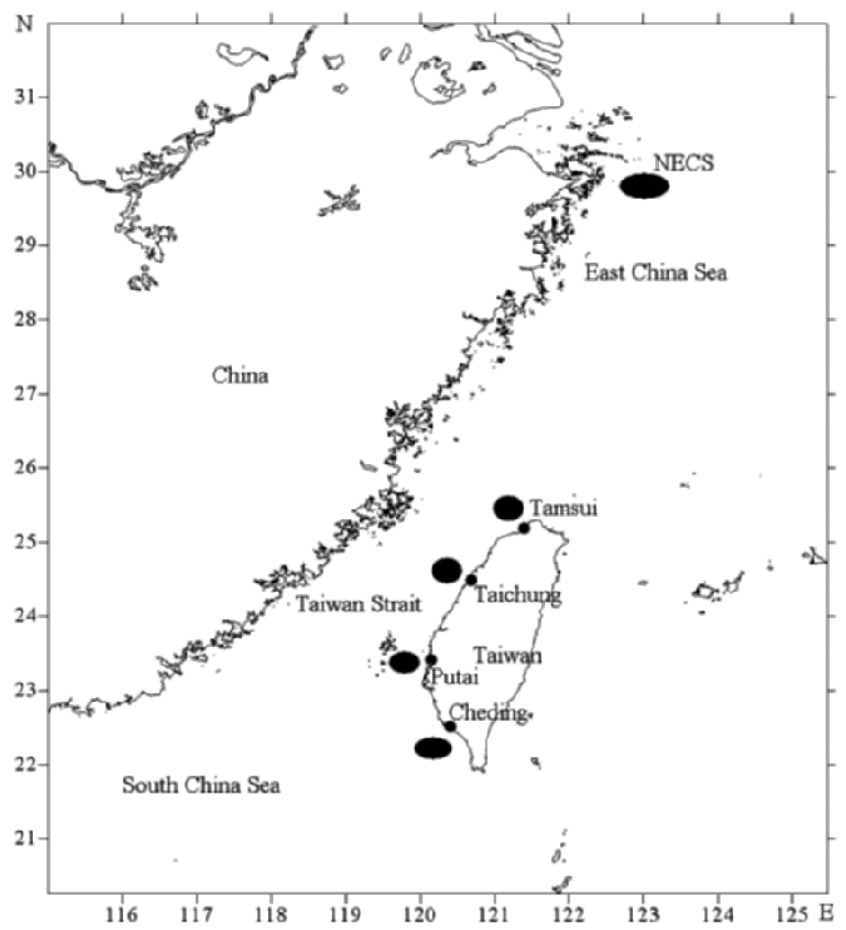

Fig. 1. Shadowed areas showing the sampling areas from the East China Sea and Taiwan Strait.

Taiwan Strait were discriminated (Tzeng, 2004). However, the variation of morphological characters could be affected by genetic and environmental factors, so that discrimination of populations based on morphological variation must be verified by genetic evidence to confirm that the variation reflects the true degree of reproductive isolation rather than environmental isolation (Pepin and Carr, 1992). In this paper, sequence analyses of the intron from the elongation factor- $1 \alpha$ gene were conducted to elucidate the population genetic structure of sword prawns in the East China Sea and Taiwan Strait.

\section{Materials And Methods}

\section{Sample Collection}

Five samples of sword prawn including 207 specimens were collected from commercial shrimp trawlers during October 2002 and February 2003 (Fig. 1 and Table 1). They were separately sampled from the north of East China Sea (NECS) and waters off Tamsui (Tamsui), Taichung (Taichung), Putai (Putai), and Cheding (Cheding). Specimens were iced or frozen immediately after capture and later kept at $-75^{\circ} \mathrm{C}$ before DNA extraction.

\section{DNA Extraction, Amplification and Sequencing}

Total genomic DNA was extracted from frozen muscle tissue using QIAamp Tissue Kit (QIAGEN) and was kept at $-20^{\circ} \mathrm{C}$ until analyses. A fragment of the intron sequence in the elongation factor- $1 \alpha$ gene was amplified using the primers EF3s (5'-GACAAGGCCCTCCGTCTTCC-3') and EF4s (5'-GGGCACTGTTCCAATACCTC-3') (France et al., 1999). PCR was run on a GeneAmp 2400 thermal cycler (Perkin-Elmer Corp.) with an initial denaturation of 60 seconds at $94^{\circ} \mathrm{C}$, followed by 30 cycles of 15 seconds at $94^{\circ} \mathrm{C}, 15$ seconds at $53^{\circ} \mathrm{C}, 15$ seconds at $72^{\circ} \mathrm{C}$, and finally a 3 -min extension at $72^{\circ} \mathrm{C}$. PCR products were visualized on an agarose gel and purified using the QIAgen PCR Purification kit. Purified PCR products were ligated into a pGEMT vector, and subsequently transformed with JM109 competent cells (Promega, Madison, USA), following manufacturer's instructions. White clones were selected from the plate. Their plasmids were prepared and then sequenced.
Table 1. Sample code, sampling locality, sample size, number of allele, and nucleotide diversity $(\pi)$ with their standard deviation (SD) in five sword prawn samples in the East China Sea and Taiwan Strait.

\begin{tabular}{llccc}
\hline \hline Sample code & \multicolumn{1}{c}{ Sampling locality } & $\begin{array}{c}\text { Sample } \\
\text { size }\end{array}$ & $\begin{array}{c}\text { Number } \\
\text { of allele }\end{array}$ & $\pi \pm \mathrm{SD}$ \\
\hline NECS & $\begin{array}{c}\text { North of the East } \\
\text { China Sea }\end{array}$ & 38 & 23 & $1.360 \pm 0.163 \%$ \\
Tamsui & Waters off Tamsui & 47 & 26 & $1.861 \pm 0.195 \%$ \\
Taichung & Waters off Taichung & 41 & 26 & $1.920 \pm 0.231 \%$ \\
Putai & Waters off Putai & 41 & 29 & $1.964 \pm 0.228 \%$ \\
Cheding & Waters off Cheding & 40 & 22 & $1.437 \pm 0.125 \%$ \\
Total & & 207 & 102 & $1.835 \pm 0.085 \%$ \\
\hline
\end{tabular}

Sequence Analyses

DNA sequences were aligned using the PILEUP program in GCG (Genetics Computer Group, version 7.0; Devereux et al., 1991). The beginning (5'GT) and end ( $\left.3^{\prime}-\mathrm{AG}\right)$ of the intron sequence were confirmed by comparing with published sequence of Penaeus vannamei Boone, 1930 (France et al., 1999). Subsequent analyses were based on the intron sequence obtained from 207 individuals. The number of variable sites was assessed with MEGA3 (Kumar et al., 2004). Each different allele was assigned a number, and the distribution of the alleles in each sampling area was determined. The nucleotide diversity $(\pi)$ (Nei, 1987) in each sample was calculated using DnaSP Version 4.10 (Rozas et al., 2003). Allele network was constructed using the median-joining method (Bandelt et al., 1999) in Network Version 4.2.0.1.

To examine whether two of the samples are genetically different from each other, the values of $F_{S T}$ statistic (Wright, 1965) between five samples were estimated and tested using the program ProSeq (Filatov, 2002). Gene flow $\left(N_{m}\right)$ was also estimated using the program ProSeq. The statistical significance of the estimate was tested through 1000 permutations. The dendrogram of five samples was constructed using unweighted pair-group method with arithmetic means (UPGMA) based on the $F_{S T}$ values with MEGA3.

Analyses of molecular variance (AMOVA) implemented in ARLEQUIN Version 2.000 (Schneider et al., 2000) were performed to test the geographic divisions among samples. Different groupings of samples were suggested by: 1) UPGMA tree of sampling areas, 2) $F_{\mathrm{ST}}$ values between samples, and 3) geographic distribution. The significance of these $\Phi$ statistics is evaluated by 1000 random permutations of sequences among samples. The groupings that maximize values of $\Phi_{\mathrm{CT}}$ and are significantly different from random distributions of individuals are assumed to be the most probable geographic subdivisions.

To check for the deviations from neutrality, Tajima's $D$ statistical test (Tajima, 1989) and Fu and Li's $D$ statistical test (Fu and $\mathrm{Li}, 1993)$ were carried out to assess evidence for population expansion using DnaSP. Population demographic history was examined by calculating mismatch distribution overall alleles with DnaSP.

\section{RESULTS}

The intron sequence from the elongation factor- $1 \alpha$ gene (206 bp in length) was identified and used for the following analyses. Nucleotide diversity $(\pi)$ was $1.835 \pm 0.085 \%$ for all samples, with values from $1.360 \pm 0.163 \%$ (NECS) to $1.964 \pm 0.228 \%$ (Putai) (Table 1). Among the 207 individuals sequenced, 102 alleles were identified. In total, 78 variable sites, including 46 singletons and 32 parsimoniously informative sites, were observed. The most common allele was observed in all samples except NECS and shared by 41 individuals; 14 specimens were from the Taichung, 10 from the Tamsui, 8 from the Putai, and 9 from Cheding samples. The second most common allele was observed in all samples and shared by 15 individuals; 1 specimen was from the Taichung, 6 from the Tamsui, 1 from the Putai, 6 from Cheding and 1 from NECS samples. The third most common allele was observed in all samples and share by 9 individuals; 1 specimen was from the Taichung, 3 from 


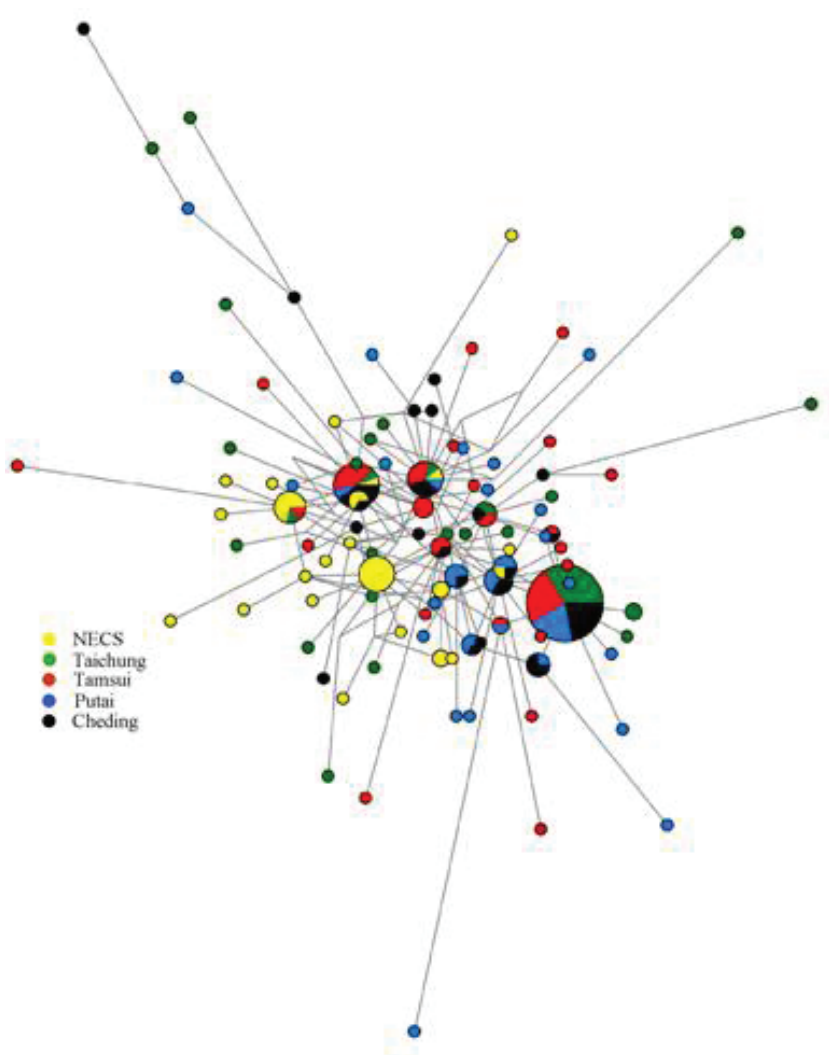

Fig. 2. Allele network of sword prawn in the East China Sea and Taiwan Strait. Different symbols represent different sampling areas. Small symbols indicate 1 individual, while large ones indicate more than 1 individual.

the Tamsui, 1 from the Putai, 3 from Cheding and 1 from NECS samples. Another allele was also shared by 9 individuals, but all these ones were from NECS sample. The median-joining network for the 102 alleles appeared star-like and showed no notable allele clustering (Fig. 2). This suggests that sword prawn probably underwent recent population expansion.

The $F_{S T}$ and $N_{m}$ values are shown in Table 2. Pairwise $F_{S T}$ values between NECS and the rest of the samples revealed significant genetic differences, but genetic variation among the other four samples were not significant. The $N_{m}$ values between all pair-wise comparisons ranged from 0.0309 (NECS-Taichung) to $\infty$ (Tamsui-Taichung). The UPGMA tree of the five is shown in Fig. 3. The five samples were clustered into two distinct groups, with NECS constituting the first group and the other four samples making up the second group.

Various groupings of samples were tested using AMOVA, but only two groupings showed significant variation (Table $3)$. In the first grouping, the AMOVA for five samples yielded a significant $\Phi_{\mathrm{ST}}$ value of 0.0827 , indicating that at least one of pair-wise comparisons revealed significant heterogeneity. In the second grouping, five samples were classified into two groups. One included NECS, the other included Tamsui, Taichung, Putai and Cheding. Significant $\Phi_{\mathrm{CT}}$ value of 0.1801 was observed, indicating that genetic discontinuity occurred in the NECS population.
Table 2. $\quad F_{S T}$ (below the diagonal) and $N_{m}$ values (above the diagonal) between five sword prawn samples in the East China Sea and Taiwan Strait. Abbreviations for sampling locations are defined in Table 1.

\begin{tabular}{lccccc}
\hline \hline & NECS & Tamsui & Taichung & Putai & Cheding \\
\hline NECS & - & 1.2408 & 0.0309 & 0.0972 & 0.9789 \\
Tamsui & $0.1677 * *$ & - & 69.2553 & 15.4259 & $\infty$ \\
Taichung & $0.1952 * *$ & $0.0036 \mathrm{~ns}$ & - & 42.0443 & 29.9052 \\
Putai & $0.2401 * *$ & $0.0159 \mathrm{~ns}$ & $0.0059 \mathrm{~ns}$ & - & 41.2861 \\
Cheding & $0.2034 * *$ & $0.0000 \mathrm{~ns}$ & $0.0083 \mathrm{~ns}$ & $0.0060 \mathrm{~ns}$ & - \\
\hline
\end{tabular}

** Significant at $P<0.001$; ns, not significant.

The model of population expansion could not be rejected because of the distribution of the pairwise number of differences in the intron alleles fitted the population expansion model well (Fig. 4). This outcome was also supported by the low Harpending's Raggedness index $(r=$ 0.0166). The significant negative values of Tajima's $D$ $(D=-2.3821, P<0.01)$ and Fu and Li's $D(D=-6.6935$, $P<0.02)$ were consistent with population expansion.

To investigate whether there was the presence of more than one locus in the elongation factor- $1 \alpha$ intron in Parapenaeopsis hardwickii, 8 additional sword prawn specimens were sequenced and compared. We sequenced 8 clones per individual. Four different sequence sizes (205, 206, 223 and 228 bp in length) were identified in 64 obtained sequences (Table 4 ). The numbers of sequences of 205, 206, 223 and 228 bp in length were 7, 47, 6 and 4, respectively. There is one specific deletion (position 199) when comparing sequences of 205 and 206 bp in length. Five deletions and 25 specific variable sites were found when comparing sequences of 223 and 228 bp in length. Number of times in which identical and different sequence were calculated when comparing two sequences of $206 \mathrm{bp}$ in length from the same individual. Multiple sequences from an individual were classified as different if they differed by at least four characters, or, when they differed by fewer than four characters, if the differences between sequences could not be attributed to polymerase error. Base the expectations under 1- and 2- locus models presented by Duda and Palumbi (1999), only one locus exists in the sequence size of $206 \mathrm{bp}$, because the number of time for identical sequence (87) is significant larger than the number of time for different sequence (31). We, therefore, concluded that there are at least four elongation factor- $1 \alpha$ loci within Parapenaeopsis hardwickii.

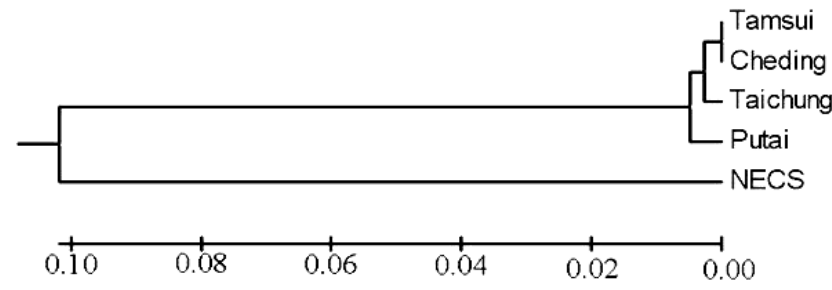

Fig. 3. UPGMA dendrogram illustrating the genetic relationships among sword prawn samples from the East China Sea and Taiwan Strait based on $F_{S T}$ values. 
Table 3. The results of AMOVA. Abbreviations for sampling locations are defined in Table 1.

\begin{tabular}{|c|c|c|c|c|}
\hline Groupings & Source of variation & Percentage of variation & $\Phi$-statistics & $P$ \\
\hline \multicolumn{5}{|l|}{ One group for all samples } \\
\hline $\begin{array}{l}1 \text { Group 1(NECS, Tamsui, Taichung, } \\
\text { Putai, Cheding) }\end{array}$ & Among locations & 8.28 & $\Phi_{\mathrm{ST}}=0.0827$ & $<0.0001$ \\
\hline \multicolumn{5}{|l|}{ Two groups } \\
\hline $\begin{array}{l}2 \text { Group } 1 \text { (NECS) } \\
\text { Group } 2 \text { (Tamsui, Taichung, Putai, } \\
\text { Cheding) }\end{array}$ & Among groups & 18.10 & $\Phi_{\mathrm{CT}}=0.1810$ & $<0.0001$ \\
\hline
\end{tabular}

\section{DISCUSSION}

Although the median-joining network for the 102 alleles revealed no genealogical branches or geographic clusters, result of cluster analysis, sequence statistic $\left(F_{S T}\right)$, and AMOVA indicated significant genetic division between the five samples. The cluster analysis indicated that the five samples could be clustered into two groups. One included the NECS sample, and the other included the other four samples (Fig. 3). $F_{S T}$ values between the NECS and the other four samples showed significant genetic differences (Table 2), indicating at least two isolated populations exist in studied area. Results of the AMOVA revealed two different populations in the East China Sea and Taiwan Strait (Table 3). Base on the above analyses, the sword prawns in the East China Sea and Taiwan Strait can be discriminated into two distinct populations. The first population is in the Taiwan Strait, and the second one in the north of the East China Sea. The present result is in agreement with the previous outcome that two morphologically distinguishable stocks separately exist in the East China Sea and the Taiwan Strait (Tzeng, 2004). Thus, to ensure resource sustainability and maintenance, the sword prawn in the Taiwan Strait and the north of the East China Sea should

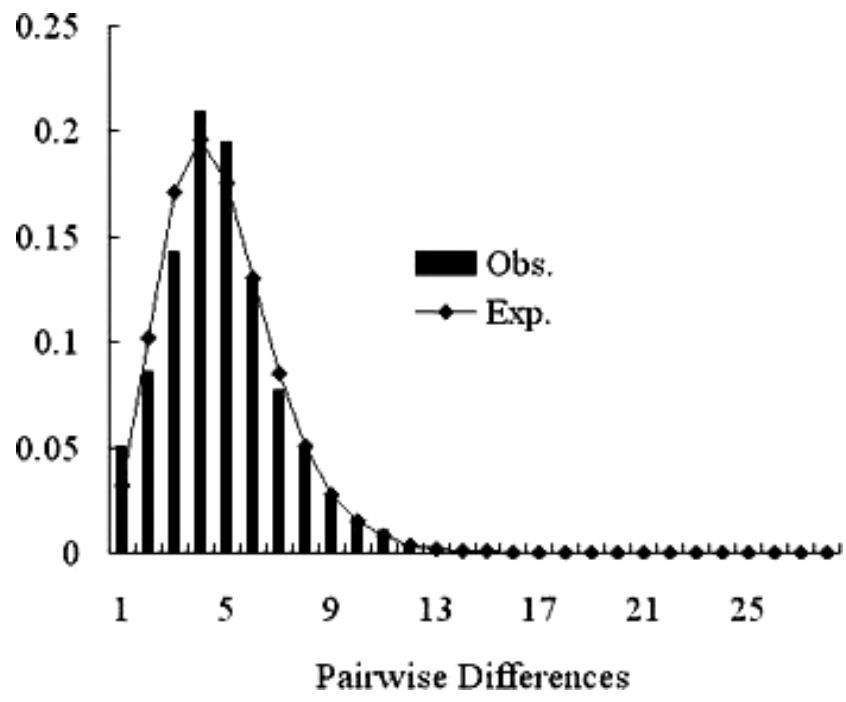

Fig. 4. Mismatch distribution constructed using pairwise differences among intron sequences of the sword prawn in the East China Sea and Taiwan Strait. be treated as two separate stocks and then be managed separately in the future.

To form and maintain discrete stocks, a moderately high degree of reproduction isolation is essential. Reproduction isolation can be developed through spawning dates, spawning frequency and spawning location. Two spawning areas were found in the Taiwan Strait and the East China Sea. One is located in the middle and north of the Taiwan Strait (Guo, 1993), but the other is located in the north of the East China Sea (Zheng and Li, 2002). In the Taiwan Strait, two peaks of spawning season were found (Guo, 1993); one is between February and April, and the other is between October and November. In the north of the East China Sea, the spawning season lasts from May to September, with the peak usually occurring between June and July (Zheng and Li, 2002). Reproductive separation in spawning date, frequency, and location may develop and keep these two discrete sword prawn stocks in the Taiwan Strait and the north of East China Sea.

Sword prawns migrate from inshore to offshore as they grow to specific size or life stage, but the migratory distance is limited (Dall et al., 1990). Thus, the dispersal of larvae is the primary source of gene flow, and ocean currents play a major role in the dispersal of this species. In the north of the East China Sea, the spawning season lasts from May to September, with the peak usually occurring between June and July (Zheng and Li, 2002). Along the eastern coast of China, the larvae of sword prawns from the north of the East China Sea may be transported to the Taiwan Strait by the China coastal current, but may not be large enough to

Table 4. Number of sequence size for 8 sword prawn specimens. Number of times in which identical or different sequence were obtained when comparing two sequences of 206 bp in length from the same individual.

\begin{tabular}{lcrcccc}
\hline \hline \multirow{2}{*}{ Specimen } & \multicolumn{7}{c}{ Sequence size } \\
\cline { 2 - 7 } 1 & $205 \mathrm{bp}$ & $\mathrm{I}^{\mathrm{a}}$ & $206 \mathrm{bp}$ & $\mathrm{D}^{\mathrm{b}}$ & $223 \mathrm{bp}$ & 228 bp \\
2 & 1 & 11 & 7 & 10 & 0 & 0 \\
3 & 1 & 9 & 7 & 12 & 0 & 0 \\
4 & 1 & 6 & 4 & 0 & 1 & 2 \\
5 & 1 & 5 & 5 & 5 & 1 & 1 \\
6 & 0 & 15 & 6 & 0 & 1 & 1 \\
7 & 1 & 15 & 6 & 0 & 1 & 0 \\
8 & 0 & 15 & 6 & 0 & 2 & 0 \\
Total & 2 & 11 & 6 & 4 & 0 & 0 \\
\hline
\end{tabular}

number of time for identical sequence. 
eliminate the genetic difference between the NECS and Taiwan Strait populations (Table 2, 3), and that is supported by lower values of $N_{m}$ between NECS and the other four samples in Taiwan Strait (from 0.0309 to 1.2408, Table 2). During the spawning season of the NECS population the China coastal current is not strong enough to flow through the Taiwan Strait, and it only spreads to the north and middle of the Taiwan Strait ( $\mathrm{Wu}, 1982)$ and results in the occurrence of sword prawn larvae mixed in the north and middle of the Taiwan Strait. This may explain why the values of nucleotide diversity in the samples from Tamsui, Taichung, and Putai are higher than ones in NECS and Cheding samples.

In the Taiwan Strait, two peaks of spawning season were found (Guo, 1993); one is between February and April, and the other is between October and November. During the late spring, the South China Sea warm water dominates the Taiwan Strait (Wang and Chern, 1989). In general, if the $N_{m}$ value is greater than about 1 , the gene flow is considered sufficient to maintain a relatively homogeneous gene pool (Slatkin, 1987). The $N_{m}$ values between all pair of four samples from Taiwan Strait are high (15.4259 to 69.2553) and prevent population differentiation between the four samples (Table 2).

Because of the fluctuation in population size, the neutrality cannot be tested accurately. The Tajima's $D$ and $\mathrm{Fu}$ and Li's $D$ statistics are sensitive to the factors such as bottlenecks or population expansion which tends to drive the values of Tajims's $D$ and Fu and Li's $D$ towards more negative values (Tajima, 1996; Martel et al., 2004). Indeed, significant negative values of these two statistics indicated that sword prawn in the East China Sea and Taiwan Strait has experienced population expansion. The unimodel mismatch frequency distribution pattern accorded well the predicted distribution under a model of population expansion (Fig. 4, Rogers and Harpending, 1992). This unimodel pattern has also been observed for other shrimp species, Farfantepenaeus aztecus Ives, 1891 and Farfantepenaeus duorarum Burkenroad, 1939 (McMillen-Jackson and Bert, 2003; 2004). Past geological and climatic events have undoubtedly played a major pole in terrestrial biogeography. During the Pleistocene glaciations, sea level was 130-150 m lower than the present level in the East China Sea and 100$120 \mathrm{~m}$ lower in the South China Sea. Consequently, the entire Yellow Sea and Taiwan Strait were exposed, and the East China Sea was reduced into an elongated trough (Wang and Sun, 1994). The disappearance of habitat had restricted marine species to the relatively limited areas and caused the mixing among populations and reduced the genetic variation between populations (Benzie and Williams, 1997). The distribution of sword prawns gradually extended corresponding to the rise of the sea level of the East China Sea and Taiwan Strait since late Pleistocene. The similar magnitude of nucleotide diversity in NECS and Cheding samples provide part of the evidence that sword prawn in the studied waters share common ancestry (Table 1).

\section{ACKNOWLEDGEMENTS}

We would like to express the gratitude for the funding supported by National Science Council, R.O.C, through contracts NSC92-2313-B-336-
001. The authors are also grateful to the reviewers' critical comments on the manuscript.

\section{REFERENCES}

Bandelt, H. J., P. Forster, and A. Rohl. 1999. Median-joining networks for inferring intraspecific phylogenies. Molecular Biology and Evolution 16: $37-48$.

Benzie, J. A. H. 1999. Genetic structure of coral reef organisms-ghosts of dispersal past. American Zoologist 39: 131-145.

- and S. T. Williams. 1997. Genetic structure of giant clam (Tridacna maxima) populations in the West Pacific is not consistent with dispersal by present-day ocean currents. Evolution 51: 768-783.

Bradley, R. D., and D. M. Hills. 1997. Recombinant DNA sequences generated by PCR amplification. Molecular Biology and Evolution 14: 592-593.

Briggs, J. S. 1999. Coincident biogeographic patterns: Indo-West Pacific Ocean. Evolution 53: 326-335.

Dall, W., B. J. Hill, P. C. Rothlisberg, and D. J. Sharples. 1990. The Biology of the Penaeidae. Academic Press, London.

Devereux, J., P. Haeberli, and P. Marquess. 1991. Genetic Computer Group Manual, version 7.0. University of Wisconsin, Madison, Wisconsin.

Filatov, D. A. 2002. ProSeq: a software for preparation and evolutionary analysis of DNA sequence data sets. Molecular Ecology note 2: 621624.

France, S. C., N. Tachino, T. F. Duda Jr, R A. Shleser, and S. R. Palumbi. 1999. Intraspecific genetic diversity in the marine shrimp Penaeus vannemei: multiple polymorphic elongation factor 1-alpha loci revealed by intron sequencing. Marine Biotechnology 1: 262-268.

Fu, Y. X., and W. H. Li. 1993. Statistical tests of neutrality of mutations. Genetics 133: 693-709.

Guo, S. Y. 1993. Growth study of sword prawn (Parapenaeopsis hardwickii) in the southwestern coast of Taiwan. Master thesis. National Sun Yat-sen University, Taiwan. [In Chinese with English abstract.]

Hellberg, M. E. 1996. Dependence of gene flow on geographic distance in two soliary corals with different larval dispersal capabilities. Evolution 50: $1167-1175$

Hillis, D. M., B. K. Mable, and C. Moritz. 1996. Molecular Systematics. Sinauer, Sunderland, Massachusetts.

Kumar, S., K. Tamura, and M. Nei. 2004. MEGA3: integrated software for Molecular Evolutionary Genetics Analysis and sequence alignment. Briefings in Bioinformatics 5: 150-163.

McMillen-Jackson, A. L, and T. M. Bert. 2003. Disparate patterns of population genetic structure and population history in two sympatric penaeid species in the southeastern United States. Molecular Ecology 12: 2895-2905.

$\longrightarrow$, and 2004. Genetic diversity in the mtDNA control region and population structure in the pink shrimp Farfantepenaeus duorarum. Journal of Crustacean Biology 24: 101-109.

Martel, C., F. Viardb, D. Bourguetc, and P. Garcia-Meuniera. 2004. Invasion by the marine gastropod Ocinebrellus inornatus in France. 1. Scenario for the source of introduction. Journal of Experimental Marine Biology and Ecology 305: 155-170.

Nei, M. 1987. Molecular evolutionary genetics. Columbia University Press, New York.

Palumbi, S. R. 1994. Genetic divergence, reproductive isolation, and marine speciation. Annual Review in Ecology and Systematics 25: 547572 .

1997. Molecular biogeography of the pacific. Coral Reefs 16: 47-52.

- and C. S. Baker. 1994. Contrasting population structure from nuclear intron sequences and mtDNA of humpback whales. Molecular Biology and Evolution 11: 426-435.

Pepin, P., and S. M. Carr. 1992. Morphological, meristic, and genetic analysis of stock structure in juvenile Atlantic cod (Gadus morhua) from the Newfoundland shelf. Canadian Journal of Fisheries and Aquatic Sciences 52: 1924-1933.

Rogers, A. R., and H. Harpending. 1992. Population growth makes waves in the distribution of pairwise genetic differences. Molecular Biology and Evolution 9: 552-569.

Rozas, J., J. C. Sanchez-DelBarrio, X. Messeguer, and R. Rozas. 2003. DnaSP, DNA polymorphism analyses by the coalescent and other methods. Bioinformatics 19: 2496-2497. 
Schneider, S., D. Roessli, and L. Excoffier. 2000. ARLEQUIN Version 2.000: A software for population genetics data analysis. Genetics and Biometry Laboratory, University of Geneva, Switzerland.

Slatkin, M. 1987. Gene flow and the geographic structure of natural populations. Science 236: 787-792.

Song, H. T., and T. M. Ding. 1993. A comparative study on fishery biology of main economic shrimps in the north of East China Sea. Journal of Zhejiang College of Fisheries 12: 240-248.

Tajima, F. 1989. Statistical method for testing the neutral mutation hypothesis by DNA polymorphism. Genetics 123: 585-595.

1996. The amount of DNA phylogeography: methods of evaluating and minimizing inference errors. Genetics 143: 1457-1465.

Tzeng, T. D. 2004. Stock identification of sword prawn (Parapenaeopsis hardwickii) in the East China Sea and Taiwan Strait inferred by morphometric variation. Fisheries Science 70: 758-764.

Wang, J., and C. S. Chern. 1989. On the Kuroshio branch in the Taiwan Strait during wintertime. Progress in Oceanography 21: 469-491.

Wang, P., and X. Sun. 1994. Last glacial maximum in China: comparison between land and sea. Catena 23: 341-353.
Wilson, A. C., R. L. Cann, S. M. Carr, M. George, U. B. Gyllensten, K. Helm-Bychowski, R. G. Higuchi, S. R. Palumbi, E. M. Prager, R. D. Sage, and M. Stoneking. 1985. Mitochondrial DNA and two perspectives on evolutionary genetics. Biological Journal of the Linnean Society 26: 375-400.

Wright, S. 1965. The interpretation of population structure by F-statistics with special regard to systems of mating. Evolution 19: 395-420.

$\mathrm{Wu}$, B. 1982. Some problems on circulation study in Taiwan Strait. Taiwan Strait 1: 1-7.

Wu, C. C. 1985. Studies on the shrimp fishery and their fishing ground in Taiwan. Bulletin of Taiwan Fisheries Research Institute 39: 169-197.

Zheng, Z. M., and M. Y. Li. 2002. Morphological and histological studies on the ovary development in Parapenaeopsis hardwickii. Journal of Fisheries of China 26: 105-109.

RECEIVED: 14 April 2007.

ACCEPTED: 17 August 2007. 